\title{
LA TERAPIA EMDR COMO TRATAMIENTO PARA EL TRASTORNO DE ESTRÉS POSTRAUMA: UNA REVISIÓN LITERARIA
}

\author{
Isaura Vanesa Gómez¹, Isabel Rosalba Gonzales ${ }^{1}$, Lina Marina Oviedo¹, Andrés Ramírez Giraldo² \\ Recibido: 19-07-2015 - Aceptado: 16-11-2015
}

\begin{abstract}
Resumen
El objetivo de este estudio es diseñar un estado del arte acerca de la terapia eye movement desensitization and reprocessing (EMDR) apartir de información publicada en la base de datos ProQuest entre los años 2013 y 2015, los resultados del estudio revelan que la terapia a pesar de que su formato original ha tenido modificaciones no deja de ser efectiva para mejorar las condiciones a nivel de salud en personas que padecen un trastorno estrés postrauma (TEPT).
\end{abstract}

Palabras clave: Trastorno por estrés postraumático (TEPT), Desensibilización y reprocesamiento a través de movimientos oculares rápidos (EMDR).

\section{EMDR THERAPY AS A TREATMENT FOR POST-TRAUMATIC STRESS DISORDER: A LITERARY REVIEW}

\begin{abstract}
The objective of this study is to design a state of the art about Eye Movement Desensitization and Reprocessing (EMDR) therapy with information found in ProQuest database in a period between 2013 and 2015. The study's results reveal that the therapy, despite being modified in its original format, keeps on being effective to improve health conditions in PTSD patients.
\end{abstract}

Key words: Posttraumatic Stress Disorder (PTSD), Eye Movement Desensitization and Reprocessing (EMDR).

${ }^{1}$ Estudiantes de psicología IX semestre de la Corporación Universitaria del Caribe -CECAR; isaura.gomezd@cecar.edu.co; Isabel. GonzalesG@cecar.edu.co; Lina.oviedoa@cecar.edu.co

${ }^{2}$ Profesor titular de la Corporación Universitaria del Caribe-CECAR; hace parte de los grupos de investigación de la institución. 
BÚSQUEDA - Julio / Diciembre de 2015 - No. 15 (51 - 63)

\section{Introducción}

El trastorno por estrés postraumático (TEPT) es el fundamento para destacar la terapia EMDR (eye movement desensitization and reprocessing, por sus siglas en inglés y en español, desensibilización y reprocesamiento a través de movimientos oculares Rápidos como técnica para el manejo del ya mencionado TEPT (Foa, Keane y Friedman, 2000). La generación de nuevas investigaciones le da peso al conocimiento científico y se constituye en una base para que los investigadores tengan un cimiento confiable y amplio para ejecutar las tareas que amerita su condición como operantes del conocimiento encontrado. Tal vez el tiempo y el mismo conocimiento hallado hasta hoy, han permitido que la temática en Psicología a nivel de trastornos mentales se multiplique, sin embargo nunca se sale de "contexto" la idea de seguir produciendo resultados en materia investigativa. Con el paso del tiempo, las costumbres pueden ir cambiando y así las culturas van sufriendo ciertas modificaciones, se necesita identificar las novedades que han surgido bien sea por el tiempo o por cualquier otro factor. Con la investigación constante acerca de los fenómenos psicológicos se actualiza el conocimiento y así el profesional gana herramientas para proporcionar mejores resultados a sus consultantes. La terapia EMDR es una técnica que ha sido flexible a los ajustes que los autores han considerado pertinentes, de acuerdo al requerimiento que tenga la situación del paciente con TEPT (Gelbach y Davis, 2007). En este caso, sería descortés hablar de la terapia
EMDR sin aportarle al lector un concepto que le permita tener claridad o por lo menos identificar qué es un TEPT; el presente trabajo pretende mostrar las investigaciones que se han publicado en los dos últimos años y que se pueden observar en la plataforma de búsqueda científica ProQuest.

\section{Metodología}

Para la realización de este estudio se hizo una revisión bibliográfica en la base de datos ProQuest acerca de terapias para el manejo del estrés publicadas entre los años 2007 y 2015, en la primera revisión se encontraron 13 resultados utilizando como palabra clave EMDR. Teniendo en cuenta que se pretendía adquirir información precisa acerca de la terapia EMDR en TEPT, se utilizó como cruce de palabras claves "EMDR and TEPT", lo que permitió descartar así 6 artículos, quedando 7 para el desarrollo del estudio, publicados entre 2013 y 2015 , los cuales cumplían a cabalidad los criterios de inclusión, los cuales exigían que estuvieran las dos palabras en los estudios a revisar.

Con la intención de que los lectores conozcan más a fondo el material utilizado para el desarrollo de éste, se presenta una tabla compuesta por los artículos seleccionados. En los resultados (ver tabla 1), se muestra cómo las diferentes investigaciones abordan el tema del TEPT y EMDR por separado y posteriormente cómo se asocian estas dos variables como procedimiento de terapia en el trastorno del TEPT. 
Isaura Gómez, Isabel Gonzales, Lina Oviedo, Andrés Ramírez G., - La terapia EMDR como tratamiento

Tabla 1. Artículos publicados entre los años 2013 y 2015 sobre EMDR y TEPT en ProQuest

\begin{tabular}{|c|c|c|}
\hline No. & Tema & Autores \\
\hline 1 & $\begin{array}{l}\text { Correlatos de la terapia EMDR en la neuroimagen funcional } \\
\text { y estructural: un resumen crítico de los hallazgos recientes. }\end{array}$ & $\begin{array}{l}\text { Marco Pagani,Högberg, G., Fernandez, I., } \\
\text { y Siracusano, A. (2015). }\end{array}$ \\
\hline 2 & $\begin{array}{l}\text { EMDR y psicosis: Pautas de conceptualización y } \\
\text { tratamiento. }\end{array}$ & $\begin{array}{l}\text { Marco Pagani, Högberg, G., Fernandez, I., } \\
\text { y Siracusano, A. (2015). }\end{array}$ \\
\hline 3 & $\begin{array}{l}\text { Terapia EMDR en el trastorno límite de personalidad/EMDR } \\
\text { therapy in borderline personality dirsorder. }\end{array}$ & $\begin{array}{l}\text { Dolores Mosquera a y Anabel González, } \\
\text { España (2013). }\end{array}$ \\
\hline 4 & $\begin{array}{l}\text { Protocolo individual de terapia EMDR para uso de } \\
\text { paraprofesionales: un estudio aleatorio controlado con } \\
\text { auxiliadores. }\end{array}$ & $\begin{array}{l}\text { Ignacio Jarero, C., Givaudan, M. y } \\
\text { Miranda, A. (2014). }\end{array}$ \\
\hline 5 & $\begin{array}{l}\text { Intervenciones humanitarias para la recuperación del } \\
\text { trauma con terapia EMDR en Latinoamérica y el Caribe. }\end{array}$ & $\begin{array}{l}\text { Ignacio Jarero, Artigas, L., Uribe, S., y } \\
\text { Miranda, A. (2015). }\end{array}$ \\
\hline 6 & $\begin{array}{l}\text { El protocolo de EMDR para incidentes críticos recientes: } \\
\text { reporte de seguimiento de su aplicación en situación de } \\
\text { masacre humana. }\end{array}$ & $\begin{array}{l}\text { Ignacio Jarero, Artigas, L., Uribe, S., y } \\
\text { Miranda, A. (2015). }\end{array}$ \\
\hline 7 & $\begin{array}{l}\text { Primer estudio de investigación de la aplicación del } \\
\text { protocolo grupal e integrativo con EMDR a niños víctimas } \\
\text { de violencia interpersonal severa. }\end{array}$ & $\begin{array}{l}\text { Ignacio Jarero,Roque-López, S., y Gómez, } \\
\text { J.,Mexico. (2014) }\end{array}$ \\
\hline
\end{tabular}

Fuente: Elaboración propia

\section{Resultados}

El TEPT según plantean Palacios y Heinze, (2002) ha sido considerada en su trabajo, como una temática relevante, hasta el punto de ocupar un lugar de importancia en los ámbitos de la investigación y el clínico. Acerca del concepto base de este estudio, el DSM V lo incluye dentro de los trastornos de ansiedad, que se diagnostica teniendo en cuenta 6 criterios (American Psychiatric Association, 2014), estos se sitúan en la tabla no. 2 .

Tabla 2. Criterios del DSM V Para los trastornos de estrés postraumático.

\begin{tabular}{|c|c|c|}
\hline \multicolumn{3}{|c|}{ Criterios del DSM V Para los Trastornos de Estrés Postraumático } \\
\hline No. & Criterios & Especificaciones \\
\hline A & $\begin{array}{l}\text { Exposición a la muerte, lesión grave o } \\
\text { violencia sexual, ya sea real o amenaza, en } \\
\text { una o más. }\end{array}$ & $\begin{array}{l}\text { - Experiencia directa del suceso(s) traumático(s). } \\
\text { - } \text { Presencia directa del(os) suceso(s) ocurrido(s) a otros(s). } \\
\text { familiar próximo o a un amigo íntimo en los casos de amenaza real } \\
\text { de muerte de un familiar o amigo, el(los) suceso(s) ha debido ser } \\
\text { violento(s) o accidental(es). } \\
\text { - Exposición repetida o extrema a detalles repulsivos del suceso(s) } \\
\text { traumático(s) (p.ej; socorristas que recogen restos humanos; } \\
\text { policías repetidamente expuestos a detalles de maltrato infantil). } \\
\text { Nota: El criterio A4 no se aplica a la exposición a través de medios } \\
\text { electrónicos, televisión, películas o fotografías, a menos que esté } \\
\text { relacionada con el trabajo. }\end{array}$ \\
\hline $\mathrm{B}$ & $\begin{array}{l}\text { Presencia de uno o más de los síntomas de } \\
\text { intrusión siguientes asociados al suceso(s) } \\
\text { traumático(s), que comienza después de un } \\
\text { suceso(s) traumático(s). }\end{array}$ & $\begin{array}{l}\text { 1. Recuerdos angustioso(s) recurrentes involuntarios e intrusivos del } \\
\text { suceso(s) traumático(s) } \\
\text { Nota: En los niños mayores de } 6 \text { años, se pueden producir juegos } \\
\text { repetitivos en los que se expresen temas o aspectos de suceso(s) }\end{array}$ \\
\hline
\end{tabular}


BÚSQUEDA - Julio / Diciembre de 2015 - No. 15 (51 - 63)

traumáticos(s)

2. Sueños angustiosos recurrentes en los que el contenido y/o al efecto del sueño está relacionado con el suceso(s) traumático(s) Nota: En los niños, pueden existir sueños alteradores sin contenidos reconocibles.

3. Reacciones disociativas (p. ej., escenas retrospectivas) en las que el sujeto siente o actúa como si se repitiera el suceso(s) traumático(s). (Estas reacciones se pueden producir de forma continua, y la expresión más extrema es una pérdida completa del entorno presente)

Nota: en los niños, la representación específica del trauma puede tener lugar en el juego.

4. Malestar psicológico intenso o prologando al exponerse a factores internos o externos que simbolizan o se parecen a un aspecto del suceso(s) traumático(s)

5. Reacciones fisiológicas intensas a factores internos o externos que simbolizan o se parecen a un aspecto del suceso(s) traumático(s).

Evitación persistente de estímulos asociados al suceso(s) traumático(s), que comienza tras el (los) suceso(s) traumático(s), como se pone de manifiesto por una o las dos características siguientes.

Alteraciones negativas cognitivas y del estado de ánimo asociadas al(los) suceso(s) traumático(s) que comienza(n) o empeoran después del(los) suceso(s) traumático(s) como se pone de manifiesto por dos o más de las características siguientes:

Alteración importante de la alerta y reactividad asociadas al(los) suceso(s) traumático(s), que comienza(n) o empeora posterior a él, como se pone de manifiesto por dos a más de las características siguientes.

1. Evitación o esfuerzos para evitar recuerdos, pensamientos o sentimientos angustiosos acerca o estrechamente asociados al(los) suceso(s) traumático(s).

2. Evitación o esfuerzos para evitar recordatorios extremos (personas, lugares, conversaciones, actividades, objetos o situaciones) que despiertan recuerdos, pensamientos o sentimientos acerca o estrechamente asociados al(los) suceso(s) traumático(s).

1. Incapacidad de recordar un aspecto importante del(los) suceso(s) traumático(s) (debido típicamente a amnesia disociaria y no a otros factores como una lesión cerebral, alcohol o drogas).

2. Creencias o expectativas negativas persistentes y exageradas sobre uno mismo, los demás o el mundo (p.ej., "estoy mal" "no puedo confiar en nadie", "El mundo es muy peligroso" "tengo los nervios destrozados".

3. Percepción distorsionada persistente de la causa o las consecuencias del(los) suceso(s) traumático(s) que hace que el individuo se acuse a sí mismo o a los demás.

4. Estado emocional negativo persistente (p.ej., miedo, terror, enfado, culpa o vergüenza).

5. Disminución importante del interés o la participación en actividades significativas.

6. Sentimiento de desapego o extrañamiento de los demás.

7. Incapacidad persistente de experimentar emociones positivas (p. ej., felicidad, satisfacción o sentimiento amoroso).

1. Comportamiento irritable y arrebatos de furia (con poca o ninguna provocación) que se expresan típicamente como agresión verbal o física contra personas u objetos.

2. Comportamiento imprudente o autodestructivo.

3. Hipervigilancia.

4. Respuesta de sobresalto exagerada.

5. Problemas de concentración.

6. Alteración del sueño (p.ej. dificultad para conciliar o continuar el sueño o sueño inquieto).

La duración de la alteración (criterios B, C, D y E) es superior a un mes.

La alteración causa malestar clínicamente significativo o deterioro en lo social, laboral u otras áreas importantes del funcionamiento.

La alteración no se puede atribuir a los efectos fisiológicos de una sustancia ( $p$. ej., medicamento, alcohol) o a otra afección médica. 
EI TEPT se puede definir como una serie de reacciones psicológicas y somáticas, debido a la vivencia de un evento que según la interpretación que se le dé, puede ser perturbador, lo que conlleva a que estas reacciones puedan aparecer en un contexto con características específicas (Baguena, 2001), en esta situación en la que el paciente está sometido por sí mismo a interpretar como perturbadores los acontecimientos, también es propenso a experimentar fuertes cambios en su funcionamiento a nivel social, ocupacional o personal (American Psychiatric Association, 1995; Ballenger, Davidson y Lecrubier, 2000); Shalev, 2000).

Vale la pena considerar que un TEPT también se puede desencadenar debido a la observación de un evento traumático que amenace la vida propia o de otras personas, el hecho de ver un evento de esta índole, puede actuar como razón para que el trastorno aparezca según lo plantean varios autores (Dongil, 2008; Iruarrizaga, Miguel-Tobal, Cano-Vindel y González-Ordi, 2004; Miguel-Tobal, Cano-Vindel, Iruarrizaga, González, y Galea, 2004; Moya-Albiol y Occhi, 2007; Olivares-Crespo, Sanz-Cortés y RoaÁlvaro, 2004); estos autores han realizado sus aportes al conocimiento sobre el trastorno, más sin embargo, es importante aclarar que en este aspecto la postura de Palacios y Heinze (2002), aunque guarda similitud en cuanto a lo que hablan sobre el trastorno también difieren un poco de esta postura; con anterioridad estos autores habían planteado que no necesariamente el observar un acontecimiento perturbador es razón única o suficiente para que aparezca el TEPT, ellos son quienes consideran que el hecho de que la persona viva un evento traumático, es una de las razones o criterios que permiten que el trastorno se desencadene. Según los mismos autores existen diferentes datos característicos de la persona tales como su historia de vida, su género, su genética, etc., que pueden o no hacer a la persona más sensible al desarrollo de un TEPT; con ello se deduce entonces que no hay regla general para desarrollar el trastorno, en gran medida o en todo su esplendor va a despender de la persona que se enfrenta al suceso traumático, entonces se deriva que el TEPT es el resultado de la combinación de la vivencia de un hecho de difícil interpretación positiva o constructiva con factores específicos individuales que hacen a la persona más susceptible a padecer una alteración de lo que ha sido su desenvolvimiento "normal" lo que permite la afectación de ese desenvolvimiento.

EI TEPT tiene la peculiaridad de marcar en un alto grado a la persona que lo sufre con el paso del tiempo, los efectos desencadenantes pueden ser diversos trayendo consigo consecuencias, como un sin número de características negativas a nivel psicológico, biológico y social para el individuo (Joseph, Williams y Yule, 1997). Como condición que afecta la salud de la persona el TEPT merece ser tratado con el objetivo de ofrecer al paciente la oportunidad de superar aquel evento que le cause cualquier tipo de daño, y que al no tratarse seguirá acarreando consecuencias en él paciente y así mismo puede generar otro tipo de trastornos como depresión, principalmente cuando ocurre en mujeres (Calvete, 2005). Desde los enfoques cognitivoconductual o cualquier otro, han sido propuestas distintas técnicas que ayudan al manejo del TEPT, como por ejemplo la restructuración cognitiva, que sin duda alguna es una técnica que revela resultados positivos, sin embrago aquí se hará énfasis en la terapia EMDR como tratamiento eficaz para el TEPT.

Basados en los resultados de la búsqueda en la base de datos ProQuest se logró evidenciar que de los años 2007 al 2015 son muy pocos las investigaciones realizadas con respecto al tema de la terapia EMDR y su tratamiento para el estrés postraumático, por lo cual se hizo necesario recopilar la información ya existente para compactarla y así dar paso a la generación y ampliación de nuevo conocimiento sobre este tema que resulta ser muy innovador y novedoso.

La terapia de reprocesamiento y desensibilización a través del movimiento ocular (EMDR) es considerada por Ehlers y cols. (2010) como un procedimiento cimentado en el convencimiento para tratar el TEPT y algunos otros de los trastornos de ansiedad. La terapia EMDR fue desarrollada por Francine Shapiro y ha sido tan efectiva que ha llegado a ser vista como una de las técnicas más eficaces para tratar entre otros casos el TEPT (Richards y Lovel, 1999). Cazabat (2004) describió las fases de la terapia EMDR, estas fases figuran a continuación en la tabla 3. 
BÚSQUEDA - Julio / Diciembre de 2015 - No. 15 (51 - 63)

Tabla 3. Fases del desarrollo de la terapia EMDR (Cazabat, 2004).

\begin{tabular}{|c|c|c|}
\hline No. & Fase & Aspectos principales \\
\hline 1 & $\begin{array}{l}\text { Historia del paciente } \\
\text { y plan de tratamiento }\end{array}$ & $\begin{array}{l}\text { Se evalúa todo tipo de aspectos que pueden influir en el tratamiento que se iniciara, como } \\
\text { las enfermedades físicas, el consumo de sustancias, desarrollo de un embarazo, etc. } \\
\text { Se concreta el objetivo del tratamiento con la intención de que el paciente este presto a } \\
\text { colaborar, facilitando la discusión de algún hecho perturbador guardado en su memoria. }\end{array}$ \\
\hline 2 & Preparación. & $\begin{array}{l}\text { Evaluación de las destrezas del paciente cuando de contrarrestar situaciones adversas se } \\
\text { trata. En ocasiones se motiva al paciente al uso de estrategias que le permitan enfrentarse } \\
\text { a esas situaciones de perturbación. }\end{array}$ \\
\hline 3 & Evaluación & $\begin{array}{l}\text { Elección de una imagen que caracterice a la situación perturbadora seleccionada, así } \\
\text { mismo se destacaran los aspectos negativos que esta genera, pensamientos, emociones } \\
\text { etc., igualmente el paciente debe sugerir un pensamiento positivo para asociarlo a la } \\
\text { interpretación negativa dada a la situación adversa. }\end{array}$ \\
\hline 4 & Desensibilización & $\begin{array}{l}\text { El paciente debe estar centrado en la situación perturbadora y las emociones y } \\
\text { pensamientos que tiene frente a ello. El terapeuta empieza el proceso, puede sugerir que } \\
\text { el paciente siga la ruta de los dedos del terapeuta mientras dialoga. } \\
\text { De igual manera el terapeuta se puede apoyar para el desarrollo de la terapia dando } \\
\text { suaves golpes en la palma de las manos o en las articulaciones de su cliente; con ayuda } \\
\text { de la audición también es posible desarrollar la terapia, permitiéndole al cliente escuchar } \\
\text { grabaciones especiales. Con todo esto se busca reprocesar los sucesos traumáticos con } \\
\text { el objetivo de modificar la interpretación dada a estos. A medida que se desarrolla la } \\
\text { terapia el terapeuta debe consultar al paciente por la obtención que ha tenido del proceso, } \\
\text { así debe esmerarse en disminuir la carga de negatividad que el paciente tenga de la } \\
\text { información reprocesada, al final la carga negativa ha debido disminuir. }\end{array}$ \\
\hline 5 & Instalación & $\begin{array}{l}\text { El paciente debe traer a su mente la cognición positiva seleccionada al inicio de la terapia, } \\
\text { así se fortifica esta. El terapeuta retoma la estimulación mientras el paciente debe recordad } \\
\text { el suceso perturbador y evaluar la cognición positiva dándole un valor de } 6 \text { o } 7 \text { en un nivel } \\
\text { de } 1 \text { a } 7 \text {. }\end{array}$ \\
\hline 6 & $\begin{array}{l}\text { Chequeo de las } \\
\text { sensaciones corporales }\end{array}$ & $\begin{array}{l}\text { Se mantiene en la mente el hecho perturbador y la cognición positiva mientras el paciente } \\
\text { recorre mentalmente por su cuerpo identificando cualquier tensión. En caso de haberla, se } \\
\text { continúa con la desensibilización. }\end{array}$ \\
\hline 7 & Cierre & $\begin{array}{l}\text { Se permite un equilibrio emocional, en caso de necesitarse alguna técnica para el manejo } \\
\text { de la ansiedad por el estado del paciente se procede a ella; se le advierte al paciente la } \\
\text { posibilidad de que en el proceso continúe mediante recuerdos o cualquier suceso que lo } \\
\text { permita en caso de que suceda se deben registrar para usarlos como herramienta para } \\
\text { lograr la salud del mismo. }\end{array}$ \\
\hline 8 & Reevaluación & $\begin{array}{l}\text { En cada sesión de la terapia se debe evaluar la situación que se trabajó en la sesión } \\
\text { anterior. Con esto se definirá si es necesario volver a trabajar esa situación en algún } \\
\text { momento. }\end{array}$ \\
\hline
\end{tabular}

Fuente: Elaboración propia

La terapia consta de ocho fases, y también dispone de tres etapas compuestas por la mismas fases (Courtois y Ford, 2009) las dos primeras fases de EMDR componen la Etapa 1, con la cual busca educar al paciente en cuanto a la terapia, se planta el objetivo, se motiva a la implementación de habilidades. Las fases 3 a la 6 conforman la atapa 2, la cual busca cambiar el inadecuado procesamiento del evento traumático y en las fases 7 y 8 de la Terapia EMDR corresponden a la Etapa 3, el cual busca aliviar y resolver la situación traumática.
La terapia EMDR fundamenta su aplicación en el inadecuado procesamiento de la información que desempeña el paciente (Shapiro 2001, 2007, citado por Mosquera y González 2011), por tanto al almacenarse en la memoria permanece en esta de manera disfuncional causándole malestar. El paciente ha hecho un procesamiento que ha afectado su salud debido a que la interpretación de las experiencias hostiles o incomodas han sido inadecuada. Para Shapiro, (1989, 2001, 2007) la génesis de los malestares clínicos se sitúa en el incongruente almacenamiento. El objetivo de la terapia EMDR es proporcionarle 
salud mental al paciente ayudándole a modificar ese almacenamiento de la información procesada de tal manera que se torne benéfico para él. La EMDR se enfoca en las experiencias del pasado sin excluir el presente que vive el paciente, como tampoco ignorando posibles sucesos futuros para minimizar los síntomas que aquejan al paciente (Jarero y Uribe, 2014); con todo esto se buscará eliminar la interpretación traumática que causa daño permitiendo ver en el sí mismo, aspectos que fortalezcan el bienestar de la persona.

El desarrollo del tratamiento EMDR comprende una composición de elementos y metodologías de diversas escuelas de terapia, entre las cuales se pueden subrayar las terapias cognitivo -conductual, técnicas de exposición, teoría de sistemas, principios freudianos, conciencia plena (Mindfulness) y terapias experienciales (Shapiro y Forest, 1997; Shapiro, Kaslow, y Maxfield, 2007). La terapia EMDR es adecuada para tratamientos que colaboren a la superación de un trauma, lo que se traduce a que requiere métodos establecidos para relacionarse con la complejidad del trastorno a tratar (Ross, 1997; Ross y Halpern, 2009; Nijenhuis y Van der Hart, 2011a; 2011b; Steele, Van der Hart, y Nijenhuis, 2009; Van der Hart, Nijenhuis, y Steele, 2006).

La base de la terapia EMDR se encuentra en el modelo de procesamiento de la linformación a estados adaptativos en Español; y en Ingles adaptive information processing (AIP) (Shapiro, 2001 citado por, Jarero, Artigas, Uribe y Miranda, 2014). El fundamento teórico de la AIP, en el cual se apoya la terapia discutida, sugiere un aporte bastante considerable en la diligencia de un tratamiento eficaz. Esta técnica tiene entre sus potencialidades el hecho de que es fácil de emplear; al terapeuta a pesar de que necesita y tiene como responsabilidad entrenarse en ella antes de llevar la aplicación a la práctica, no le resulta compleja de desarrollar y además sus derivaciones son ligeras.

En el estudio realizado por Jarero, et al. (2014) se describe que la EMDR en su validez, es aceptada por La Organización Mundial de la Salud (2013) y otras entidades internacionales para el tratamiento de TEPT en cualquier etapa de la vida desde la niñez, pasando por la juventud y aún en la adultez (Bisson y Andrew, 2007). Lo anterior supone una ventaja debido a que en cualquier etapa del desarrollo humano la persona puede experimentar un evento estresante y puede potencialmente desarrollar un TEPT. Autores como Wadda, Zaharim y Alqashan (2010) valoraron la presencia de la sintomatología del TEPT en niños que emigraron hacia Malasia para escapar de la guerra que se vivía en Irak; con su estudio los autores hallaron que el $68.5 \%$ de la población padecía la sintomatología para TEPT, 12 de los niños que presentaban los síntomas, los cuales tenían edad entre 7 y 12 años con el consentimiento de sus tutores recibieron 12 consultas de terapia EMDR. Antes de iniciar el proceso, se definieron 2 grupos de los cuales 1 era para someterse a tratamiento y el otro no. Antes de iniciar las sesiones de EMDR se evaluó el nivel de TEPT en ambos grupos y no se halló diferencia relevante; posterior a la aplicación de la terapia se volvió a evaluar el mismo aspecto en ambos grupos y la diferencia en esta segunda evaluación fue significativa para el grupo intervenido.

En el año 2008 autores como Jarero, Artigas y Montero, revelaron los resultados de su investigación acerca de la tragedia vivida en México en el año 2006, como resultado del desplome de una mina en el país azteca hubo 65 mineros muertos, por lo cual el personal de un equipo de AMAMECRISIS se desplazó hasta la zona del suceso para apoyar a los niños que presentaban trauma al igual que duelo por el evento vivido. En este caso 16 niños que perdieron a sus padres (hombres) fueron participes de la terapia EMDR-IGTP. Después de la aplicación de la terapia, se estudiaron los efectos de la misma y se halló que el puntaje de la Escala CRTES (Child's Reaction to Traumatic Events Scale) mostró una desvalorización significativa de la sintomatología traumática. En 2007, Oh y Choi, realizaron un estudio SPECT EMDR con 2 pacientes que padecían trauma por accidente de tránsito. Después de la aplicación de EMDR los investigadores hallaron una mejora en el estado de los pacientes con trauma desde beneficios cerebrales. 
BÚSQUEDA - Julio / Diciembre de 2015 - No. 15 (51 - 63)

La EMDR sugiere que se deben eliminar las experiencias que el paciente ha vivido y que por la interpretación que se le está dando, ya que está causando malestar en la persona, así mismo se tiene en cuenta la situación del aquí y el ahora, para contribuir al buen estado del paciente en el futuro (Shapiro, 2001, 2006).

Las ventajas de la terapia no finalizan en que sea aplicable a niños, jóvenes y/o adultos, además se puede aplicar a un campo más amplio donde sea necesario tratar de situaciones perturbadoras (Shapiro, 2001; Solomon y Shapiro, 2008), este planteamiento evidencia la oportunidad de desarrollar EMDR con un paciente que atraviese cualquier etapa de su vida y como no puede faltar, que padezca un TEPT; son entonces 2 criterios indiscutibles para aplicar la terapia al paciente. El EMDR se puede emplear con personas que presenten síntomas psicóticos guarden relación con eventos perturbadores pasados. Un ejemplo que plantean Hardy, et al., (2005) y Morrison, et al., (2003) manifiesta como ejemplo una paranoia que tuvo sus inicios inmediatamente después de un suceso traumático como un atraco o bien sea los sonidos que se asocian al trauma. Cuando los eventos de la vida ejercen influencia secretamente en la psicosis gracias a las creencias centrales o hipótesis acerca de sí mismo y el resto de personas, es viable aplicar EMDR para reducir los síntomas psicóticos. El hecho de haber tenido experiencia negativas en la infancia como resultado de la interacción con las demás personas puede generar en el futuro expectativas negativas hacia el resto de la sociedad, desarrollándose síntomas paranoides (Fisher et al., 2012).

Otras de la aplicaciones de la terapia EMDR y quizá la que más se ha discutido como tratamiento es los sufrimientos clínicos apoyados en vivencias estresantes (Shapiro, 2001; Solomon y Shapiro, 2008). Otra evidencia que fundamenta la idea de que la EMDR es aplicable para el tratamiento TEPT es el planteamiento de la APA (2004) y Bisson y Andrew (2007).

Hasta el momento quizá el lector ha podido identificar que la aplicabilidad de la terapia es efectiva para la reducción de la sintomatología traumática, por ello se busca dejar claro las diferentes modalidades de aplicación de EMDR. La terapia y TEPT ha pasado por procesos de modificación que permiten su diligencia individual y/o grupal según sea necesario, es por ello que Jarero, Artigas y Luber (2011) declaran que de cualquier manera en que sea usada la terapia resulta efectiva para el paciente. Adler y Settle (2008) y Fleming (2012) describen la efectividad de EMDR desde su desarrollo individual precisamente con pacientes con sintomatología traumática en niños; de igual manera algunos autores han aplicado lo que se conoce como el protocolo grupal e integrativo (EMDR-IGTP), Artigas, Jarero, Alcalá y López (2014) han aplicado un modelo grupal de EMDR combinando el formato inicial de la terapia constituido por ocho fases y además una técnica conocida como el abrazo de la mariposa, lo cual ha resultado completamente efectivo (Artigas y Jarero, 2014).

Al revisar mayor información científica se ha identificado que EMDR y las terapias de exposición tienen resultados clínicos positivos en el tratamiento del TEPT (Davidson y Parker, 2001). Además de tratar la perturbación presente de un evento reciente específico, optar por aplicar aspectos de la terapia aun sin haberse presentado los síntomas del TEPT es un avance especial para disminuir las posibilidades de guardar información que contenga una carga perturbadora y que conduzca a la indebida interpretación de los acontecimientos (Tofani y Wheeler, 2011).

Es importante destacar que además de las adaptaciones que se han mencionado anteriormente al modelo original de EMDR, también la investigación ha permitido exponer EMDR-PRECI (incidentes críticos recientes), este nuevo ajuste resulta apropiado si se busca disminuir la sintomatología del TEPT en la etapa adulta (Jarero et al., 2011; Jarero y Uribe 2011, 2012). Siguiendo la misma línea de destacar los ajustes de la terapia, existe EMDR-PROPARA o el protocolo individual de terapia de reprocesamiento y desensibilización a 
través del movimiento ocular para para uso de profesionales en situaciones de trauma agudo, este es un modelo aplicado para profesionales más específicamente personas que trabajan bajo contrato o voluntarios, que otorgan servicios críticos en emergencias; como lo son: policías, operadoras de líneas de emergencia, bomberos, personal de rescate y búsqueda, personal de ambulancias y personal de salas de urgencias; que al igual que los demás modelos propuestos hasta este punto cumple con las expectativas de mejoramiento (Jarero, Amaya, Givaudán y Miranda, 2013).

\section{Conclusiones}

Los estudios que fundamentan este material de investigación convergen en que la Terapia EMDR es efectiva en cuanto al tratamiento de TEPT, permitiéndole al paciente superar el evento traumático; modificando la interpretación que ha tenido del mismo, no obstante diferentes autores han optado por realizar modificaciones, en este caso nuevos protocolos, al momento de aplicar EMDR lo que no necesariamente afecta el diseño original del autor principal pero si encierra dentro de sí un enfoque de entrenamiento en la terapia para una población especial. Siguiendo en la misma discusión se subraya que la es terapia efectiva pese a los agregados que le hacen algunos autores lo cual fundamenta en mayor medida sus efectos.

Al tomar como fundamento las diferentes modalidades que hoy día caracterizan a EMDR resulta posible a la imaginación manifestar que quizá en algunos años al revisar los estudios que se hagan se encuentre mayor número de modalidades de enseñanza o de ejecución de la misma con respecto a situaciones tan específicas de aplicación como ya algunos autores han hecho. Lo que conlleva a la fusión de la EMDR con otras técnicas de igual magnitud para garantizar en mayor medida los resultados positivos de la intervención, EMDR hasta el momento ha sido tan flexible a los ajustes que ha tenido que sin exasperarse se puede deducir que probablemente con el tiempo vengan nuevas modificaciones.
Igualmente a pesar de ser una técnica que tiene éxito como tratamiento y con resultados rápidos en el TEPT, en Colombia a pesar de todas las víctimas por los diferentes conflictos que existen, no se ha hecho ningún estudio publicado en los últimos 8 años alrededor de esta temática, sería interesante ver si ese impacto también se observa en nuestra población o al menos aumentar los referentes de éxito o no de este procedimiento terapéutico.

\section{Referencias bibliográficas}

Adler-Tapia, R. L., y Settle, C. S. (2008). EMDR and the art of psychotherapy with children. New York, NY: Springer.

American psychiatric association, (1995). Manual diagnóstico y estadístico de los trastornos mentales., 4ta edición. Washington: Masson.

American psychiatric association. (2004). Practice guidelines for the treatment of patients with acute stress disorder and posttraumatic stress disorder. Arlington, VA: Author.

American psychiatric association. (2014). Diagnostic and statistical manual of mental disorders (5th ed). Washington, DC: Author. http://dx.doi.org/10.1891/19333196.8.4.E143

Artigas, L., Jarero, I., Alcalá, N., y López Cano, T. (2014). The EMDR integrative group treatment protocol (IGTP) for children. In M. Luber (Ed.), Implementing EMDR early interventions for man-made and natural disasters (pp. 237-251). New York, NY: Springer Publishing.

Artigas, L., y Jarero, I. (2014). The butterfly hug. In M. Luber (Ed.), Implementing EMDR early mental health interventions for manmade and natural disasters (pp. 127-130). New York, NY: Springer Publishing.

Báguena Puigcerver, M. J. (2001). Tratamientos psicológicos eficaces para el estrés post- 
BÚSQUEDA - Julio / Diciembre de 2015 - No. 15 (51 - 63)

traumático. Psicothema, 13(3) 479-492.

Ballenger J, Davidson J, Lecrubier Y (2000). Focus on posttraumatic stress disorder. J Clin Psychiatry, 61 (supl 5): 3-12.

Bisson, J., y Andrew, M. (2007). Psychological treatment of post-traumatic stress disorder (PTSD). Cochrane Databaseof Systematic Reviews, 18(3): CD003388.

Calvete, E. (2005). Género y vulnerabilidad cognitiva a la depresión: el papel de los pensamientos. Ansiedad y Estrés, 11, 203214. http://pendientedemigracion.ucm.es/ info/seas/comision/tep/tep/index.htm

Cazabat, E. (2004) EMDR: alivio rápido y duradero para una variedad de problemáticas. Casos clínicos. V Congreso Virtual de Psiquiatría. http://www.emdr-es.org/prensacont1.htm

Courtois, C., y Ford, J.D. (2009). Treating complex traumatic stress disorders. An evidencebased guide. New York: Guilford Press.

Davidson, P. R., y Parker, K. C. H. (2001). Eye movement desensitization and reprocessing (EMDR): A meta-analysis. Journal of Consultant Clinical Psychology, 69, 305-316.

Dongil-Collado, E. (2008). Reestructuración cognitiva: un caso de estrés postraumático. Ansiedad y Estrés, 14, 265-288. http:// pendientedemigracion.ucm.es/info/seas/ comision/tep/tep/index.htm

Donguil-Collado, E (2009). Trastorno por estrés postraumático. Ansiedad y Estrés.

Ehlers, A., Bisson, J., Clark, D. M., Creamer, M., Pilling, S., Richards, D., Yule, W. (2010). Do all psychological treatments really work the same in posttraumatic stress disorder? Clinical Psychology Review, 30, 269-276.

Fisher, H.L Schreier, A., Zammiit, S., Maugham, B, Munafó, M. R., Lewis, G., y Wolke, D. (2012) Pathways between childhood victimization and psychosis-like symptoms in the ALPAC birth cohort. Schizophrenia Bulletin, 39, 1045- 1055.

Fleming, J. (2012). The effectiveness of eye movement desensitization and reprocessing in the treatment of traumatized children and youth. Journal of EMDR Practice and Research, 6(1), 16-26.

Foa, E.B., Keane, T.M., y Friedman, M.J. (2000). Effective treatments for PTSD. Practice guidelines from the international society for traumatic stress studies. New York: Guilford.

Gelbach, R., y Davis, K. (2007). Disaster response: EMDR and family systems therapy under community wide stress. In F. Shapiro, F. W. Kaslow, y L. Maxfield (Eds.), Handbook of EMDR and family therapy processes (pp. 387-406). New York, NY: Wiley.

Hardy, A., Fowler, D., Freeman, D., Smith, B., Steel, C., Evans, J, Dunn, G. (2005). Trauma and hallucinatory experience in psychosis. The Journal of Nervous and Mental Disease, 193(8), 501-507

Iruarrizaga, I., Miguel-Tobal, J. J., Cano-Vindel, A., y González-Ordi, H. (2004). Consecuencias psicopatológicas tras el atentado terrorista del 11-M en Madrid en víctimas, familiares y allegados. Ansiedad y Estrés, 10, 195206. http://pendientedemigracion.ucm.es/ info/seas/comision/tep/tep/index.htm

Jarero, I., Amaya, C., Givaudan, M., y Miranda, A. (2014). Protocolo individual de terapia EMDR para uso de paraprofesionales: Un estudio aleatorio controlado con auxiliadores. Journal of EMDR Practice and Research, 8(4), E156-E166. Recuperado de http://search.proquest.com/ docview/1629985362? accountid=34487

Jarero, I., Artigas, L., Uribe, S., y Miranda, A. (2014). EMDR Ttherapy humanitarian trauma recovery interventions in latin America and the caribbean. Journal of EMDR Practice and Research, 8(4), 260- 
Isaura Gómez, Isabel Gonzales, Lina Oviedo, Andrés Ramírez G., - La terapia EMDR como tratamiento

268.

Jarero, I., Artigas, L., Uribe, S., y Miranda, A. (2015). Intervenciones humanitarias para la recuperación del trauma con terapia EMDR en Latinoamérica y el caribe. Journal of EMDR Practice and Research, 9(2), E69-E78. Recuperado de http://search.proquest.com/ docview/1676460491?accountid=34487

Jarero, I., Artigas, L., y Luber, M. (2011). The EMDR protocol for recent critical incidents: Application in a disaster mental health continuum of care context. Journal of EMDR Practice and Research, 5(3), 82-94.

Jarero, I., Artigas, L., y Montero, M. (2008). The EMDR integrative group treatment protocol: Application with child victims of a mass disaster. Journal of EMDR Practice and Research, 2, 97-105.

Jarero, I., Roque-López, S., y Gómez, J. (2014). Primer estudio de investigación de la aplicación del protocolo grupal e integrativo con EMDR a niños víctimas de violencia interpersonal severa. Journal of EMDR Practice and Research, 8(4), E143-E155. Recuperado de http://search.proquest.com/ docview/1629985441?accountid=34487

Jarero, I., y Uribe, S. (2011). The EMDR protocol for recent critical incidents: Brief report of an application in a human massacre situation. Journal of EMDR Practice and Research, 5(4), 156-165.

Jarero, I., y Uribe, S. (2012). The EMDR protocol for recent critical incidents: Follow-up report of an application in a human massacre situation. EMDR Practice and Research, 6(2), 50-61.

Jarero, I., y Uribe, S. (2014). Worst case scenarios in recent trauma response. In $\mathrm{M}$. Luber (Ed.), Implementing EMDR early mental health interventions for man-made and natural disasters (pp. 533-541). New York,
NY: Springer Publishing.

Jarero, I., y Uribe, S. (2014). Worst case scenarios in recent trauma response. In M. Luber (Ed.), Implementing EMDR early mental health interventions for man-made and natural disasters (pp. 533-541). New York, NY: Springer Publishing.

Joseph, S., Williams, R. y Yule, W. (1997): Understanding post-traumatic stress. A psychosocial perspective on PTSD and treatment. New York. Wiley.

Miguel-Tobal, J.J., Cano-Vindel, A., Iruarrizaga, I., González, H., y Galea, S. (2004). Consecuencias psicológicas de los atentados terroristas del 11-M en Madrid. Planteamiento general de los estudios y resultados en la población general. Ansiedad y Estrés, 10, 163-179. http:// pendientedemigracion.ucm.es/info/seas/ comision/tep/tep/index.htm

Morrison, A. P., Frame, L., y Larkin, W. (2003). Relationships between trauma and psychosis: A review and integration. The British Journal of Clinical Psychology/The British Psychological Society, 42(Pt. 4), 331-353.

Mosquera, D. y González A. (2011). Del apego temprano al TLP [From early attachment to BPD]. Revista Mente y Cerebro, 18-27.

Mosquera, D., y González, A. (2013). Terapia EMDR en el trastorno límite de personalidad/EMDR therapy in borderline personality disorder. Acción Psicológica, 10 (1), 85-95. Recuperado de http://search.proquest.com/ docview/1460987474? accountid=34487

Moya-Albiol, L. y Occhi, S. (2007). Sintomatología del Trastorno por Estrés Postraumático y empatía en población no directamente afectada tras los atentados terroristas de Madrid del 11 de Marzo de 2004. Ansiedad y Estrés, 13, 269-281. http:// pendientedemigracion.ucm.es/info/seas/ 
BÚSQUEDA - Julio / Diciembre de 2015 - No. 15 (51 - 63)

comision/tep/tep/index.htm

Nijenhuis, E.R.S., y Van der Hart, O. (2011a). Dissociation in trauma: A new definition and comparison with previous formulations. Journal of Trauma and Dissociation, 12, 416- 445.

Nijenhuis, E.R.S., y Van der Hart, O. (2011b). Defining dissociation in trauma. Journal of Trauma and Dissociation, 12, 469-473.

Oh, D. H., y Choi, J. (2007). Changes in the cerebral perfusion after EMDR. Journal of EMDR Practice and Research, 1, 24-30.

Olivares-Crespo, M. E., Sanz-Cortés, A., y RoaÁlvaro, A. (2004). Trastorno de estrés postraumático asociado a cáncer: revisión teórica. Ansiedad y Estrés, 10, 43-61. http://pendientedemigracion.ucm.es/info/ seas/comision/tep/tep/index.htm

Pagani, M., Högberg, G., Fernandez, I., y Siracusano, A. (2015). Correlatos de la terapia EMDR en la neuroimagen funcional y estructural: Un resumen crítico de los hallazgos recientes. Journal of EMDR Practice and Research, 9(2), E58-E68. Recuperadodehttp://search.proquest.com/ docview $/ 1676460306$ ?accountid $=34487$

Ross, C.A. (1997). Dissociative identity disorder. Diagnosis, clinical features, and treatment of multiple personality (2nd ed.). New York: John Wiley.

Ross, C.A., y Halpern, N. (2009). Trauma model therapy. A treatment approach for trauma, dissociation, and complex comorbidity. Richardson, TX: Manitou Communications.

Shalev Ay (2000). Post traumatic stress disorder: diagnosis, history, and life course. En: Post-traumatic stress disorder diagnosis, management and treatment. Nutt $D$, Davidson JRT, Zohar J (eds.), pp. 1-15. Martin Dunitz Ltd,

Shapiro, F. (1989). Efficacy of the eye movement desensitization procedure in the treatment of traumatic memories. Journal of Traumatic Stress Studies, 2, 199-223.

Shapiro, F. (2001). Eye movements, desensitization and reprocessing: Basic principles, protocols, and procedures (2nd Ed.). New York, NY: Guilford Press.

Shapiro, F. (2006). EMDR: New notes on adaptive information processing, with case formulation principles, forms, scripts, and worksheets. Watsonville, CA: EMDR Institute.

Shapiro, F. (2007). EMDR and case conceptualization from an adaptive information processing perspective. En F. Shapiro, F. Kaslow y L. Maxfield (Eds.), Handbook of EMDR and family therapy processes (pp. 3-36). New York: Wiley.

Shapiro, F. (2007). EMDR, adaptive information processing, and case conceptualization. Journal of EMDR Practice and Research, 1, 68-87.

Shapiro, F., Kaslow, F.W. y Maxfield, L. (2007). Handbook of EMDR and family therapy processes. New York: John Wiley.

Shapiro, F., y Forrest, M. (1997). EMDR. The breakthrough therapy for overcoming anxiety, stress and trauma. New York: Basic Books.

Solomon, R. M., y Shapiro, F. (2008). EMDR and the adaptive information processing model: Potential mechanism of change. Journal of EMDR Practice and Research, 2(4), 315325.

Steele, K., Van der Hart, O., y Nijenhuis, E.R.S. (2009). The theory of trauma-related structural dissociation of the personality. In P.F. Dell y J.A. O'Neil (Eds.), Dissociation and the dissociative disorders: DSM-V and beyond (pp. 239-258). New York: Routledge.

Tofani, L. R., y Wheeler, K. (2011). The recenttraumatic episode protocol: Outcome 
Isaura Gómez, Isabel Gonzales, Lina Oviedo, Andrés Ramírez G., - La terapia EMDR como tratamiento

evaluation and analysis of three case studies. Journal of EMDR Practice and Research, 5(3), 95-110.

Van den Berg, D.,P.G., Van der Vleugel, B.,M., Staring, A. B. P., De Bont, P.,A.J., y De Jongh, A. (2015). EMDR y psicosis: Pautas de conceptualización $\mathrm{y}$ tratamiento. Journal of EMDR Practice and Research, 9(1), E29-E45. Retrieved from http://search.proquest.com/ docview $/ 1652867040$ ?accountid $=34487$

Van der Hart, O., Nijenhuis, E.R.S., y Steele, K. (2006). The haunted self: Chronic traumatization and the theory of structural dissociation of the personality. New York: Norton
Van der Kolk, B.A., McFarlane, A.C. y Wisaeth, L. (eds.) (1996): Trau - matic stress. The effects of overwhelming experience on mind, body, and society. New York. The Guilford Press.

Wadda, N. N., Zaharim, N. M., y Alqashan, H. F. (2010). The use of EMDR in treatment of traumatized Iraqi children. Digest of Middle East Studies, 19(1), 26-36.

World Health Organization. (2013). Guidelines for the managements of conditions specifically related to stress. Geneva, Switzerland: Author. 